\title{
048 \\ RADIATION EXPOSURE REDUCTION DURING TRANSCATHETER AORTIC VALVE IMPLANTATION (TAVI)
} PROCEDURES

D Sharma, A Ramsewak, S O'connaire, R Verghis, G Manoharan, M S Spence Royal Victoria Hospital

doi:10.1136/heartjnl-2013-304019.48

Introduction Trans-catheter aortic valve replacement (TAVR) is a new treatment for symptomatic aortic stenosis in patients who are considered high risk or inoperable for conventional surgical aortic valve replacement. The radiation dose from this procedure has not been extensively investigated. The methods to keep these doses as low as possible should be actively investigated for the safety of both the patient and the medical team involved.

Aims In this single centre analysis, we established our radiation dose and determined whether lower fluoroscopy and cine imaging settings would reduce patient and operator doses while maintaining an acceptable image quality.

Methods Data on radiation exposure was collected for consecutive patients undergoing TAVI. Using a single plane C-arm, the settings were changed from coronary to electrophysiology (EP), this resulted in a reduction for fluoroscopy, from 15 pulse-progressive fluoroscopy (ppf) to $3.75-7.5 \mathrm{ppf}$ and for cine acquisition, from 15 frames per second (fps) to 3.75-7.5 fps for access site management. We compared the radiation doses in this lower settings (LS) group with the standard settings (SS) group.

Results Data for 68 consecutive patients was collected over a period of 11 months from the Royal Victoria Hospital, Belfast. The median dose area product using the standard setting was $10210 \mathrm{cGy} \mathrm{cm}{ }^{2}$ as compared to $6928 \mathrm{cGy} \mathrm{cm}^{2}$ for the lower setting group was statistically significant ( $p$ value 0.006 ). The screening time, procedure time and contrast volume in the two groups were not statistically different. There were no procedural deaths or radiation burns in either group at 30 days follow-up.

Conclusions This study demonstrates that it is possible to reduce radiation dose by approximately $32 \%$ by adhering to radiation safety protocols, employing protective measures and lowering cine-fluoroscopy imaging settings, while preserving acceptable image quality. 